\title{
The Role of Cultural Capital Sports Oriented on Workers' Participation in the Newspaper in Tehran, Iran
}

\author{
Gholizadeh Abbas \\ Commercial and Advertising Manager, Keyhan Printing \\ Institute, Tehran, Iran \\ Gholizadeh_ab@yahoo.com \\ Mehraein Sepideh \\ Ph.D. Candidate Sport Science \\ Department of Sports Studies, Faculty of Educational \\ Studies \\ University Putra Malaysia \\ sepideh20_k@yahoo.com
}

\author{
Kaveh Khabiri \\ Ph.D., SportsPhysiology, AssistantProfessor and \\ FacultyMember \\ Faculty of Humanities, Islamic Azad University of \\ Ashtian, Arak, Iran \\ sepka_kh@yahoo.com \\ Aida Mehrad \\ Ph.D., Social Psychology \\ Faculty of Psychology \\ Universitat Autonoma de Barcelona \\ aida_238@yahoo.com
}

\begin{abstract}
This study examined the role of cultural capital sport oriented on workers' participation. The active presence of workers in different workplaces is very significant; additionally, consider their physical health is so imperative. Moreover, distinguishing the effective factors that can increase the amount of workers' physical health and persuade them to exercise is essential; also, lack of attention to them leads to several difficulties. In the study, focused on $\mathbf{4 0 0}$ workers who worked for the newspaper in Tehran, Iran. One questionnaire for assessing demographic factors and cultural capital sport oriented were used. Findings of the study showed that cultural capital sport oriented have significant relationship with workers' participation. Also, body image determined as the first reason for sport among workers. In overall, the sport has a noticeable role in workers' life and it can grow their mind and body health. In this regard, the head of workers should be developed some methods for introducing workers to the sport; likewise, determined valuable programs for them to increase physical power and growing muscles.
\end{abstract}

Keyword: Cultural Capital, Physical Education, Sport, Workers' Participation

Received 18 October 20I7/Accepted 30 November 2017 @ JEHCP All rights reserved 


\section{Introduction}

Sport and physical education assumed as one of the vital features in modern life. In fact, the physical education determined as a training and educational process that involved procedure of motor skills, fitness, and the advance of positive mind; likewise, it is caused of the flourishing of the individuals in sport. In reality, these goals do not specify for a particular age and include all the individuals. Considering a sport as normal behavior that cause of health and happiness is significant; moreover, most of the health centers pay attention to this performance behavior. In the other word, the sport is an imperative activity that accomplished in different styles in various cultures and societies. This human activity has a noticeable role in healthy body and mind; in fact, it can decline mental stress. Furthermore, considering to the sport and performing it based on society level has been emphasized, significantly (Fathi, 2004). In the same vein, sociologists have been deliberated to the culture and activate that occurred on it. Also, based on cultural psychology perspective, the content of a culture can be useful for behavior and performance of community members (Berry, Poortinga, Segal, \& Dasen, 2003).

\section{Cultural Capital Sport Oriented}

In the cultural area, there are numerous debates that have been explained by sociologists and psychologists. One of these famous researchers were Jurkowski, Mosquera, and Ramos (2010) that described the cultural factors and illustrated their relation to physical activity. Similarly, Vosoughi and Khosravinezhad (2009) have examined the relationship between cultural and social factors on emotional behavior of football fans. The researchers clarified that cultural and social factors have a brilliant role in emotional behaviors.

Regarding sociological perspective, sport in cultural aspect originated from social class; and individuals based on their life and cultural level choose their favorite sport (Coakley \&White, 1992; Moienoldini \& Sanatkhah, 2013). This view is supported by Fathi (2004) who emphasized on sport based on cultural aspect and explained its relation with ethnicity and race. Cultural capital can be effective in selecting sports-oriented lifestyle. In another word, the cultural capital 
associated with knowledge and education; as well, in during of sociability illustrates educational and skills capability as valuable cultural goals. The cultural capital assumed as one of the main sport behavioral factors amongst various groups in society. In fact, this factor included different tendencies that appeared in during the process of socialization (Bourdieu, 1988). The culture can pass amongst individuals, ordinary; and in some cases, it breaks customs and laws, and appears in a different vision. Accordingly, there is a cultural identity that individuals based on this factor introduced themselves. The cultural identity comprised various items such as language, religion, cultural geography, history and cultural memory, and social system. In Iran same as other societies, there is specific cultural identity; and categorized in language, customs, celebrations, believes, modernity, etc. Based on Young, Harris, and Mabry (2002) providing more cultural facilities in life environment has a noticeable role in increasing physical activity. Similarly, Wilbur, Chandler, Dancy, Choi, and Plonczynski (2002) emphasized the important role of cultural factors in physical exercise. In effect, cultural factors are crucial in motivation and physical performance (Thompson et al., 2002). Considering to physical and mental health is the main factor for tending to the sport; on the other hand, cultural limitations are caused by ignoring physical and mental health (Fathi, 2004).

In general, focusing on the role of cultural capital sport oriented and its subordinates (body image, fashions, and media) with workers' participation in the newspaper, Tehran context remains remarkably inadequate. Whereas sport assumed as one of the practical factors in every society that can improve individuals' performance and behavior. In this regard, professionally focusing on sport based on different views is considerable. Till now, the researchers just focused on sport in a various society that performed by an individual, not via the impact of some external factors such as cultural. Accordingly, the present study is conducted to fill in the actual literature gap. 


\section{Method}

Overview

This study focused on quantitative approach to the research objective and determines the relationship between research variables (cultural capital sport oriented and participation of workers). Likewise, this study used the cross-sectional design and concentrated on a sample at one point in time.

\section{Participants}

The sample size of the current study was determined based on Cochran's sample size formula. Regarding this formula has been examined 400 workers in newspaper offices in Tehran, Iran.

\section{Measurement}

The questionnaire of this study included two parts (demographic and cultural capital sport oriented) that were devised by the researcher. The questionnaire included 29 items and measures demographic factors, body image, fashions, and media. In this questionnaire, for the part of cultural capital sport oriented used 5-point Likert scale $(5=$ completely agree to I=completely disagree).

\section{Data analysis}

Descriptive statistics and t-test have been used for analyzing the data via SPSS version 20 statistical software.

\section{Results}

Table I shows a descriptive statistic of cultural capital sport oriented and its subordinates. In this Table illustrates the cultural capital sport oriented in overall with a mean value of $4.2 \mathrm{I}(S D=0.497)$. Also, body image with a mean value of $4.59(S D=0.59 \mathrm{l})$ has the highest mean among subordinates of cultural capital sport oriented, while fashions with an average value of 
$3.86(S D=0.7 / 8)$ and media with a mean value of $4.18(S D=0.778)$ have the lowest mean among cultural capital sport oriented subordinates.

Table I: Descriptive Statistic of Cultural Capital Sports Oriented and Its Subordinates $(\mathrm{N}=400)$

\begin{tabular}{|c|c|c|c|}
\hline Variable & & $\bar{M}$ & SD \\
\hline \multirow{4}{*}{$\begin{array}{l}\text { Cultural capital } \\
\text { sport oriented }\end{array}$} & & 4.21 & 0.497 \\
\hline & Body Image & 4.59 & 0.591 \\
\hline & Fashions & 3.86 & 0.718 \\
\hline & Media & 4.18 & 0.778 \\
\hline
\end{tabular}

$\mathrm{M}=$ Mean/SD=Standard Deviation

In Table 2 illustrates body image $t(399)=4.59, p=.00 \mathrm{I}$, fashion $t(399)=3.86, p=.00 \mathrm{I}$, and media $t(399)=4.18, p=.00 \mathrm{I}$. Also workers' participation $t(399)=15.28, p=.00 \mathrm{I}$. Additionally, Cohen's effect size $(d=0.50)$ recommended a moderate significance.

Table 2: The Results of Cultural Capital Sport Oriented Factors and Workers participation based on t-test $(\mathrm{N}=400)$

\begin{tabular}{cccc}
\hline \hline Variable & $\mathrm{t}$ & $\mathrm{df}$ & $\mathrm{P}$ \\
\hline Workers Participation & 15.28 & 399 & $0.00 \mathrm{I}$ \\
Body Image & 4.59 & 399 & 0.001 \\
Fashions & 3.86 & 399 & 0.001 \\
Media & 4.18 & 399 & 0.001 \\
\hline \hline
\end{tabular}

Thus, the results showed that there is a significant difference between cultural capital sport oriented subordinates and workers' participation. Also, the cultural capital sport oriented plays 
a brilliant role in motivating workers' participation and increasing their healthy body and mind. Additionally, the body image likewise determined as the main reason for sport and physical exercise amongst workers.

\section{Discussion}

Regarding the findings of the study and the meaningful role of cultural capital sport oriented, can say that body image as one of the main subordinates has an imperative role in workers' view and performance. Additionally, the body image has more mean value in cultural capital sport oriented. On the other hand, fashion and media do not have high mean value as compared with body image based on workers' attitude. As well, the results demonstrated the cultural capital sport oriented and its subordinates (body image, fashion, and media) have a meaningful association with workers' performance. In reality, cultural capital sport oriented has a considerable role in activity and effective present of workers at the workplace. The findings are in parallel with Wilbur, Chandler, Dancy, Choi, and Plonczynski (2002) who focused on physical activity and explained that external factors such as culltural factors play considerable role on individuals' performance; Fathi (2004) likewise emphasized on cultural items on performance and social activities of individuals. The researcher clarified cultural items assumed as positive factors on individuals' activities that appear in their personal and social life. In this regard, Vosoughi and Khosravinezhad (2009), and also Jurkowski, Mosquera, and Ramos (2010) explored cultural factors that impacted on attitude and performance of individuals. They described cultural factors have considerable impact on individuals' activity specifically in sport areas; in reality, there are meaningful relationship between cultural factors and activities of individuals.

\section{Conclusion}

Sport plays the main role in individuals' lives, and it helps to improve and growth body and mind. In this regard, sociologists and psychologists endeavor to obtain wide and adequate 
knowledge in the sports area. Also, they want to connect society and sport to gather. Based on the results, the relationship between cultural capital sport oriented subordinates and workers' participation were significant; also, the body image assumed mainly factor as compared to fashion and media. Based on these consequences, society and supervisors have a considerable responsibility toward workers and their performance. In some societies there is not enough knowledge for offering accurate ways about the sport and its impacts on body and mind; additionally, providing suitable atmosphere amongst the workers for exercise. Then, it is necessary the society and supervisors improve the policies about exercise and motivate workers' attitude toward the sport; likewise, award them about advantages of exercise and physical movement.

\section{Reference}

Berry, J.W., Poortinga, H.Y., Segal, M. H. \&Dasen, R. R. (2003). Cross-cultural psychology research and affliction. $2^{\text {nd }}$ ed. Cambridge, Cambridge university press.

Bourdieu, P. (1988). Program for a sociology of sport. Sociology of Sports Journal, 5(2), I 53-I6I.

Fathi, S. (2004).Study the effective cultural and social factors on sports tendency among studentathletes. (Unpublished master's thesis). Islamic Azad University of Tehran, Iran.

Jurkowski, J., Mosquera, M. \& Ramos, B. (2010). Selected cultural factors associated with physical activity among Latino women. Women's Health Issues, 20(3), 219-226.

Coakley, J.\& White, A. (1992). Making decision: Gender and sports participation among British adolescents. Sociology of sports journal, 9, 20-35.

Moienoldini, J.\& Sanatkhah, A. R. (20I3). Socio-cultural factors influencing citizens to the sport (case study: Kerman). Urban Studies, 3, I78-I49.

Thompson, J., Allen, P., Cunningham-Sabo, L., Yazzie, D., Curtis, M.\&Davis, S. (2002). Environmental, policy, and cultural factors related to physical activity in sedentary American Indian women. Women \& Health, 36(2), 57-72.

Vosoughi, M. \&Khosravinezhad, S. (2009). Investigating of the social-cultural factors of effective on the excitement behavior of football watchers. Social sciences bulletin, 3(I), I I7-I 40. 
Journal of Educational, Health and Community Psychology

Vol 6, No 3, 20I7, E-ISSN 2460-8467

Abbas, Khabiri, Sepideh, Mehrad

Wilbur, J., Chandler, P., Dancy, B., Choi, J.\& Plonczynski, D. (2002). Environmental, policy, and cultural factors related to physical activity in urban, African American women. Women \& Health, 36(2), 17-28.

Young, D. H, X., Harris, J. \& Mabry, I. (2002). Environmental, policy, and cultural factors related to physical activity in well-educated urban African American women. Women \& Health, 36(2), 29-4I. 